\title{
Correction to: Laparoscopic gastrectomy and metastasectomy for stage IV gastric cancer
}

\author{
Sa-Hong Min ${ }^{1,4} \cdot$ Yongjoon Won ${ }^{1} \cdot$ Kanghaeng Lee $^{1} \cdot$ Sang II Youn ${ }^{1} \cdot$ Guowei Kim ${ }^{1,3} \cdot$ Young Suk Park $^{1}$. \\ Sang-Hoon Ahn ${ }^{1,2}$. Do Joong Park ${ }^{1,2}$. Hyung-Ho Kim ${ }^{1,2}$ (1)
}

Published online: 12 May 2020

○) Springer Science+Business Media, LLC, part of Springer Nature 2020

\section{Correction to: Surgical Endoscopy} https://doi.org/10.1007/s00464-020-07592-7

This article was updated to correct Do Joong Park's name:

Do Joong (given name) Park (family name).

Publisher's Note Springer Nature remains neutral with regard to jurisdictional claims in published maps and institutional affiliations.

The original article can be found online at https://doi.org/10.1007/ s00464-020-07592-7.

Hyung-Ho Kim

hhkim@snubh.org

1 Department of Surgery, Seoul National University Bundang Hospital, 82, Gumi-ro 173 beon-gil, Bundang-gu, Seongnam-si 13620, Gyeonggi-do, Republic of Korea

2 Department of Surgery, Seoul National University College of Medicine, Seoul, Republic of Korea

3 University Surgical Cluster, National University Health System, Singapore, Singapore

4 Present Address: Chungnam National University Sejong Hospital, Daejeon, South Korea 